\title{
Current Modulation by Optoelectric Control of Ferroelectric Domains
}

\author{
Gaurav Vats,* Jani Peräntie, Jaakko Palosaari, Jari Juuti, Jan Seidel,* and Yang Bai* \\ Cite This: ACS Appl. Electron. Mater. 2020, 2, 2829-2836 \\ Read Online
}

ABSTRACT: Optoelectric control of domains is likely to pave the foundation for optoferroelectric devices. This work reports the combined effect of light and lowvoltage electric bias for optoelectric control of ferroelectric domains in a semiconducting ceramic material-KNBNNO $\left(\left(\mathrm{K}_{0.5} \mathrm{Na}_{0.5}\right) \mathrm{NbO}_{3}\right.$ doped with 2 mol \% $\left.\mathrm{Ba}\left(\mathrm{Ni}_{0.5} \mathrm{Nb}_{0.5}\right) \mathrm{O}_{3-\delta}\right)$. The effect is utilized to achieve two orders of magnitude amplification in electrical response, asymmetric AC modulation, and domain velocities of $30000 \mathrm{~nm} \mathrm{~s}^{-1}$ with ultralarge domain switching areas of over $30 \mu \mathrm{m}$ in fractions of a second. The charge injection due to light illumination on this material causes the tuning of material conductivity and acts as a virtual

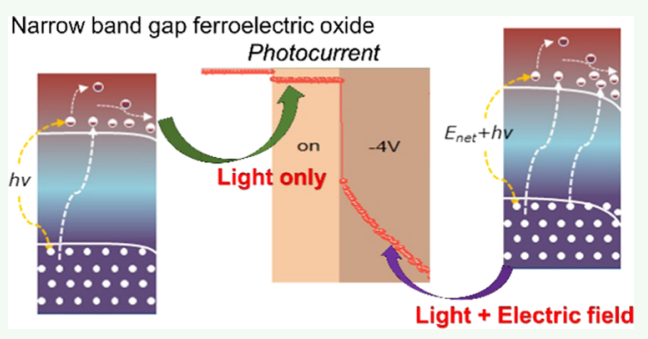
electrode. Based on this mechanism, a proof of concept for a monolithic ferroelectric light-effect transistor with a source and drain as electrical contacts with light acting as a virtual gate is demonstrated. This is likely to offer a potential solution to the scaling limit of conventional three-terminal transistors. The same device is also demonstrated to work as a photodiode, a half-wave rectifier, and an electrical output modulator.

KEYWORDS: KNBNNO, photoferroelectric, photo-induced ferroelectricity, optical virtual electrode, optoferroelectric

\section{INTRODUCTION}

The renaissance of light-ferroelectric interactions has attracted significant research interest in recent years ${ }^{1-4}$ due to advancement in the understanding of underlying mechanisms. $^{5-9}$ This has resulted in renewed interests in enhanced ferroelectric light energy conversion with large efficiency, ${ }^{10}$ and the development of new concepts such as domain wall photovoltaics, ${ }^{8,9}$ tip-enhanced photovoltaic effects, ${ }^{11}$ and shift current models. $^{5-7}$ Parallel ongoing investigations of the possibility of utilizing optical control of ferroelectric domains ${ }^{12-15}$ for the tuning of capacitance/resistive states, ${ }^{16-18}$ multiferroic states, ${ }^{19}$ and macroscopic polarization $^{13,14}$ have also opened new pathways for the creation of next-generation neuromorphic devices such as photodetectors, ${ }^{20}$ optical modulators, ${ }^{21}$ ferroelectric diodes, ${ }^{22}$ memristors, ${ }^{18}$ solaristors, ${ }^{23}$ optoelectric tunnel junctions, ${ }^{24,25}$ and photoferroelectric generators. ${ }^{2,26,27}$ Some commercial devices for optoelectronic applications of ferroelectrics are based on $\mathrm{LiNbO}_{3}$, with other materials being less explored. ${ }^{28-34}$ In this work, a cost-effective and easy-to-fabricate polycrystalline bulk ferroelectric (KNBNNO: $\left(\left(\mathrm{K}_{0.5} \mathrm{Na}_{0.5}\right)\right.$ $\left.\left.\mathrm{NbO}_{3}-2 \mathrm{~mol} \% \mathrm{Ba}\left(\mathrm{Ni}_{0.5} \mathrm{Nb}_{0.5}\right) \mathrm{O}_{3-\delta}\right)\right)^{14,17,35}$ that has a $60 \%$ lower electro-optic poling energy requirement than $\mathrm{LiNbO}_{3}$ crystals $^{28-34}$ is presented.

KNBNNO is a band gap-engineered $\left(E_{\mathrm{g}}=1.6 \mathrm{eV}\right)$ ferroelectric ceramic that has been reported as a monolithic multifunctional energy conversion material with above band gap photovoltages. ${ }^{35}$ These alluring features make it intriguing for several electronic and energy harvesting devices ${ }^{2,36}$ and nanoscale optoelectric investigations. ${ }^{37}$ The material has been documented for reversible optical control of ferroelectric domains with domain velocities of the order of $0.01 \mathrm{~nm} \mathrm{~s}^{-1}$, corresponding to domain wall displacements of $\sim 20 \mathrm{~nm}$ in 15 min under the influence of a continuous laser source (wavelength: $405 \mathrm{~nm}$; intensity: $8.3 \mathrm{~W} \mathrm{~cm}^{-2}$ ). ${ }^{14}$ To be viable for practical applications, the optical switching time and domain wall velocities need to be increased significantly.

To address the issue, this work utilizes the cumulative effect of light and low electric fields. First, a correlation in electrical and optical behavior of KNBNNO is established and an understanding of the mechanism of optoelectric control of ferroelectric domains is developed. The switched domain areas under the cumulative effect of the light and electric field are found to be controlled by the applied voltage and laser spot size. Light illumination is found to act as a virtual electrode, and the charge injection/modulation in electrical output is governed by the ferroelectric photovoltaic effect and optical tuning of the material conductivity. The same phenomenon is verified macroscopically, and modulation of the photocurrent as well as the electrical current is demonstrated. Guided by these findings, applications of fast ultralarge domain switching,

Received: June 11, 2020

Accepted: August 7, 2020

Published: August 7, 2020 
a ferroelectric function generator, direct current (DC) amplification, and asymmetric modulation of alternate current (AC) are achieved. Based on these findings, KNBNNO is found to work as a monolithic (in a metal-ferroelectricsemiconductor-metal (MFSM) parallel plate configuration) solaristor, ${ }^{23}$ phototransistor, ${ }^{23}$ photo field-effect transistor (FET),$^{38}$ photoswitch, ${ }^{39}$ and a light-effect transistor (a device with two electrical terminals and light acting as a virtual gate: same as a parallel plate capacitor). ${ }^{40}$

\section{EXPERIMENTAL SECTION}

KNBNNO ceramic samples were synthesized via solid-state route from mixed reactants of $\mathrm{K}_{2} \mathrm{CO}_{3}, \mathrm{Na}_{2} \mathrm{CO}_{3}, \mathrm{BaCO}_{3}, \mathrm{NiO}$, and $\mathrm{Nb}_{2} \mathrm{O}_{5}$. The reactants were calcined at $850{ }^{\circ} \mathrm{C}$ for $4 \mathrm{~h}$ and subsequently pressed green bodies were sintered at $1165{ }^{\circ} \mathrm{C}$ for $2 \mathrm{~h}$. The sintered samples were polished to a $100-120 \mu \mathrm{m}$ thickness with an average surface roughness of $50-60 \mathrm{~nm}$. It should be noted that careful control of the stoichiometry is needed to ensure the repeatability of the material fabrication and desired functionality. This is well known for all perovskite compositions containing alkaline elements, and it is even more critical for the KNBNNO where minority doping is present. The influence of the stoichiometry on microstructures and functions of KNBNNO has been discussed in previous works. ${ }^{35,41}$ The concentration of oxygen vacancy, i.e., the $\delta$ value, may be tuned with the sintering atmosphere like argon, nitrogen, and oxygen to compensate any possible deviation of the stoichiometry thus the overall functionality. Figure $S 1$ in the Supporting Information presents the quality of the KNBNNO ceramic sample, where the Xray diffraction (XRD) pattern and energy-dispersive $\mathrm{X}$-ray spectroscopy (EDXS) mapping confirm the correct KNBNNO composition and orthorhombic perovskite phase, ${ }^{35,41}$ and the sharp XRD reflections and scanning electron microscopy (SEM) image show the high crystallinity of the grains and the high density of the polycrystalline sample. ${ }^{35}$

For nanoscale measurements, the samples were further polished to a surface roughness of $5-10 \mathrm{~nm}$ on one side and this surface was scanned under atomic force microscopy (AFM) and piezoresponce force microscopy (PFM) with a $405 \mathrm{~nm}$ laser source. The other surface was coated with a $\mathrm{Cr}-\mathrm{Au}$ electrode for bottom conductive contact.

For macroscopic measurements, two sample and electrode configurations were prepared. Configuration 1: the fabricated samples were coated with $200 \mathrm{~nm}$ thick indium tin oxide (ITO) electrodes on both sides. The coated samples were then laser-cut into small rectangular (1-2 mm length and width) and round (1-2 $\mathrm{mm}$ diameter) samples. This configuration was used for out-of-plane measurement. Configuration 2: small-sized rectangular (1-2 mm length and width) Ag electrodes were deposited on the same surface of the sample through masked doctor blading. The gaps between the electrodes ranged from 100 to $200 \mu \mathrm{m}$. This configuration was used for in-plane measurements. During the high field $\left(6 \mathrm{kV} \mathrm{mm}^{-1}\right)$ poling, each sample was immersed in silicone oil at room temperature with the poling voltage on for $30 \mathrm{~min}$ in the dark. The photocurrent was measured, and the external electric field was provided by the SourceMeter (2450, Keithley) connected with a probe station (PVX400, Wentworth Laboratories, UK). The probes were coated with $\mathrm{Au}$ and directly contacted with the ITO electrodes on the sample, acting as the pickup electrode of electrical signals. The light source was a $405 \mathrm{~nm}, 50 \mathrm{~mW}$ laser (OBIS LX/LS Series, Coherent), of which the beam spot size was $0.8 \pm 0.1 \mathrm{~mm}$ in diameter at $1 / e^{2}$. Further experimental details are given in the Supporting Information.

\section{ANALOGY IN OPTICAL AND ELECTRICAL BEHAVIOR}

First, an understanding of the analogies between the electrical and optical behavior of KNBNNO was developed at the nanoscale on an unpoled sample. Figure $1 \mathrm{a}-\mathrm{c}$ shows the PFM

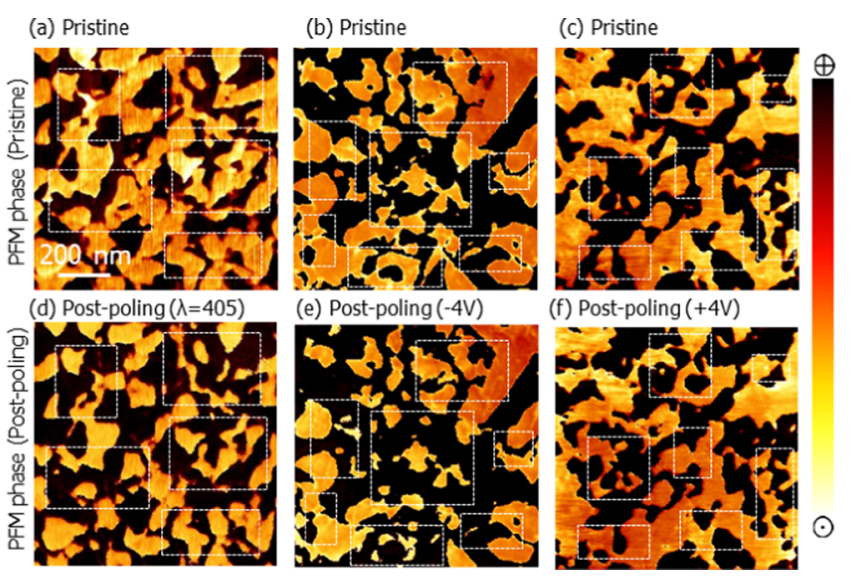

Figure 1. Comparison of electrical and optical response: $(a-c)$ PFM out-of-plane phase images of ferroelectric domains at three different locations in a dark condition. PFM images of ferroelectric domains (d) post optical poling with a laser source of $405 \mathrm{~nm}$ wavelength with no electrical bias, post electrical poling with (e) $-4 \mathrm{~V}$ (electric field $-40 \mathrm{~V} \mathrm{~mm}^{-1}$ ) in the dark and $(\mathrm{f})+4 \mathrm{~V}$ (electric field $+40 \mathrm{~V} \mathrm{~mm}^{-1}$ ) in the dark. All images were acquired on an unpoled sample. The sample was kept in the dark for over $2 \mathrm{~h}$ before each measurement to avoid the effect of partial poling due to ambient white light exposure. All measurements were carried out with the configuration, where $\mathrm{Cr}-\mathrm{Au}$ was the bottom electrode and the PFM tip acted as the top electrode.

out-of-plane phase images of ferroelectric domains at three different locations captured in the dark. Figure $1 \mathrm{~d}$ displays the post optical poling (with zero DC electric bias) domain structure of KNBNNO, which was acquired after $15 \mathrm{~min}$ of continuous laser exposure, showing that the bright domains (up polarization) have shrunk due to laser exposure. Figure S2 in the Supporting Information confirms the reversibility of the process, i.e., bright domain regions tend to recover after turning off the laser. The domain orientation was altered under illumination, while it recovered after the illumination was removed. Such a nonremanent effect was because the electric field induced by the surface charge carriers generated under illumination was not strong enough to overcome the potential barrier to permanently switch the domain orientation, i.e., it happened on the linear part of the ferroelectric hysteresis loop. ${ }^{14,17,42}$ All the optical measurements were performed with a light source of $405 \mathrm{~nm}$ wavelength (laser spot size: $0.06 \mathrm{~mm}^{2}$; power: $5 \mathrm{~mW}$; intensity: $8.3 \mathrm{~W} \mathrm{~cm}^{-2}$ ). This incident light was chosen because in our previous study ${ }^{14}$ we found that although KNBNNO is sensitive to the full visible range, the maximum outputs/changes in the ferroelectric domains are observed with the wavelength of $405 \mathrm{~nm}$. In general, the change of macroscopic polarization in KNBNNO increases with the incident light photon energy and power. ${ }^{14}$ As has been studied in the previous research, ${ }^{43}$ the alteration and reversal of ferroelectric domains under illumination may involve electronic polarization strengthening of the ionic polarization vector in the direction of the applied electric field.

Figure 1e,f reveals the post electrical poling (both performed in the dark) behavior with $-4 \mathrm{~V}$ (electric field $-40 \mathrm{~V} \mathrm{~mm}^{-1}$ ) and $+4 \mathrm{~V}$ (electric field $+40 \mathrm{~V} \mathrm{~mm}^{-1}$ ), respectively. A shrinkage of bright domains within the negatively poled area is observed (Figure 1e), while the bright domains were expanded due to positive poling, as shown in Figure 1f. This indicates that the light and negative bias work in an analogous manner and support the switching of ferroelectric domains in an 


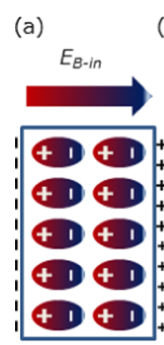

(d)

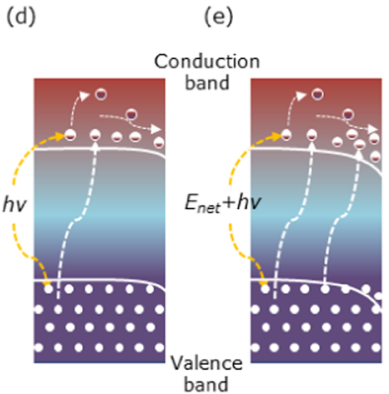

(c)
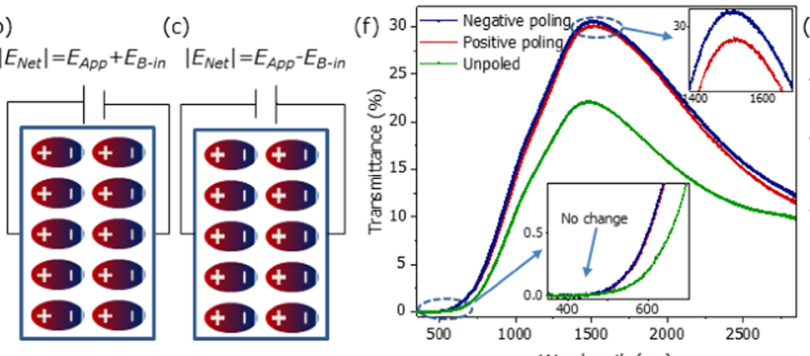

Wavelength $(\mathrm{nm})$

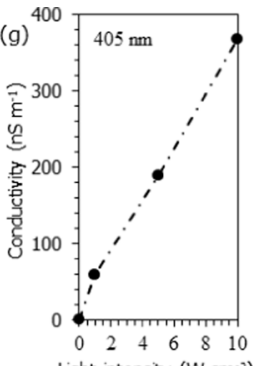

Light intensity $\left(\mathrm{W} \mathrm{cm}^{-2}\right)$
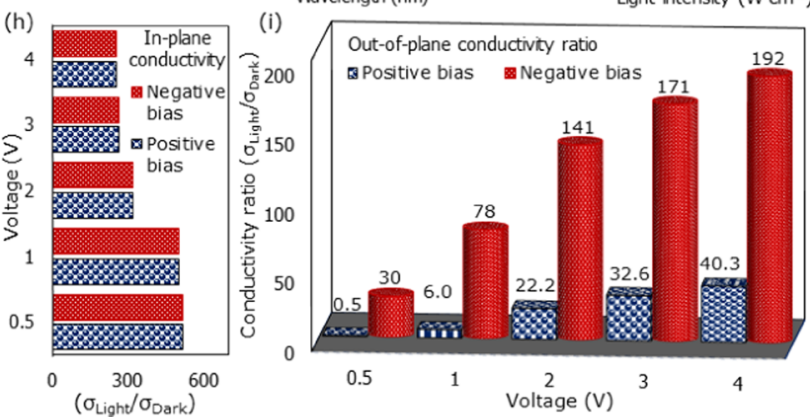

Figure 2. Material properties under the application of positive and negative electric fields: (a) built-in field ( $\left.E_{\mathrm{B} \text {-in }}\right)$ due to the polarization of the material. Effect on external bias applied $\left(E_{\mathrm{App}}\right)$ in the $(\mathrm{b})$ same and $(\mathrm{c})$ opposite direction of the built-in field. The schematic of charge migration from the valence to the conduction band in the presence of (d) light only and (e) under the cumulative effect of the light and electric field. The cumulative effect of the light and electric field tilts the band profile and gives rise to a drift of the excited carriers. (f) Transmission spectra of unpoled, positively poled, and negatively poled samples with ITO electrodes on both surfaces. $(\mathrm{g})$ Change in sample conductivity along with sample thickness as a function of the laser intensity. The ratio of the light and dark electrical conductivities measured as a function of applied DC bias (from \pm 0.5 to $\pm 4 \mathrm{~V}$ ) along the $(\mathrm{h})$ in-plane direction in an unpoled sample in ferroelectric semiconductor field-effect transistor configuration ${ }^{45}$ with an inter-electrode gap of $140 \mu \mathrm{m}$ and (i) out-of-plane direction in a poled $120 \mu \mathrm{m}$ thick KNBNNO sample sandwiched between ITO electrodes in MFSM configuration. Note: the light penetration depth for KNBNNO was $200 \mu \mathrm{m}$. (g, i) Out-of-plane device configuration and the (h) in-plane device configuration.

identical fashion. The phenomenon was further confirmed by ruling out the effect of temperature change due to laser exposure $^{14}$ and by comparing the photocurrent and electrical current (in the dark), ${ }^{14}$ measured using a conductive AFM tip of $30 \mathrm{~nm}$ in radius. The effect of light-assisted localized heating and the pyroelectric effect can also be ruled out based on the experimental evidence of a nominal change $\left(<2 \mu \mathrm{C} \mathrm{cm}^{-2}\right)$ in remanent polarization over a broad temperature range of $20-$ $150{ }^{\circ} \mathrm{C}$ (observed by macroscopic polarization-electric field $(P-E)$ loops shown in our previous work on KNBNNO) ${ }^{14}$ All nanoscale experiments were performed at room temperature and the localized temperature change corresponding to the wavelength of $405 \mathrm{~nm}$ was found to be only $0.5{ }^{\circ} \mathrm{C}$ (direct measurement using a thermocouple). ${ }^{14}$ Therefore, it has been concluded that the light-dependent changes in ferroelectric domains are primarily due to the compensation of lightinduced charges on the sample surface. ${ }^{14}$ Figure S3a in the Supporting Information shows the growth (100 s) and decay $(250 \mathrm{~s})$ of the photocurrent. The dark electrical current responses to the voltages of $-1 \mathrm{~V}$ (electric field $-10 \mathrm{~V} \mathrm{~mm}^{-1}$ ) and $+1 \mathrm{~V}$ (electric field $+10 \mathrm{~V} \mathrm{~mm}^{-1}$ ) are presented in Figure $S 3 b, c$ in the Supporting Information, respectively. Although the growth and decay times for the photocurrent and electric current $(1 \mathrm{~s}$ each) are different, the same direction of the photocurrent and electrical current with $-1 \mathrm{~V}$ supports the argument of analogy in the photoresponse and an applied negative bias on the sample. The variation in the magnitude of electrical response for $+1 \mathrm{~V}\left(18 \mathrm{~mA} \mathrm{~cm}^{-2}\right)$ and $-1 \mathrm{~V}(-28 \mathrm{~mA}$ $\mathrm{cm}^{-2}$ ) indicates an asymmetry in the device configuration. This induced asymmetry could be due to the difference in the electrode area or the work function difference between the two electrodes (30 nm Pt AFM tip on one side while a $4 \times 4 \mathrm{~mm}^{2}$ $\mathrm{Au}-\mathrm{Cr}$ electrode on the other side).

The same domain areas in Figure $1 \mathrm{a}, \mathrm{d}, \mathrm{b}, \mathrm{e}, \mathrm{c}, \mathrm{f}$, respectively, were identified using the topography images. Reproducibility of the results was confirmed through repeated measurements at different locations also as can be evident from Figures S5S11 in the Supporting Information.

\section{LIGHT AS A VIRTUAL ELECTRODE}

It is important to note that any asymmetry in the state of polarization leads to a built-in field in the material, resulting in a change in the density of bound charge carriers, as shown in Figure 2a. The applied electric field will either be supported or opposed by this built-in field, which will govern the magnitude of the indicated net applied electric field $\left(E_{\mathrm{Net}}\right)$, as shown in Figure $2 b, c$. Due to this reason, it is often difficult to reliably measure the doping polarity and charge carrier density in ferroelectrics. However, it is evident that ferroelectric photoresponse depends on band-to-band or intra-band excitations and is explained using ballistic and/or shift current models. ${ }^{1,2,5,10,44}$ The effect further depends on the externally applied field, and the photoconductivity and photovoltaic effects (including the bulk photovoltaic effect and the Schottky interface effect). ${ }^{2}$ The applied electric field in addition to the resultant built-in field due to laser exposure leads to modification of the Fermi-level and tilts the band gap profile, as shown in the schematic of Figure 2d,e. Figure $2 d$ shows that in the absence of an applied electric field, the light illumination helps in the excitation of charge carriers from the valance to the conduction band, which are collected at the electrode due to the presence of the built-in field. The applied electric field 

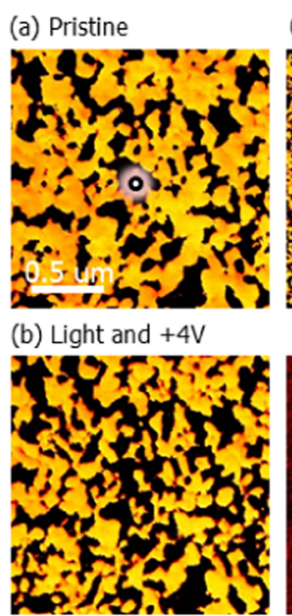

(d) Pristine

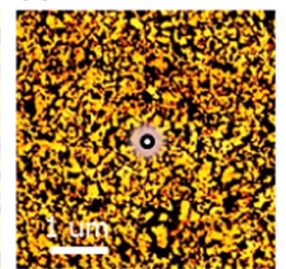

(e) Light and $-4 \mathrm{~V}$
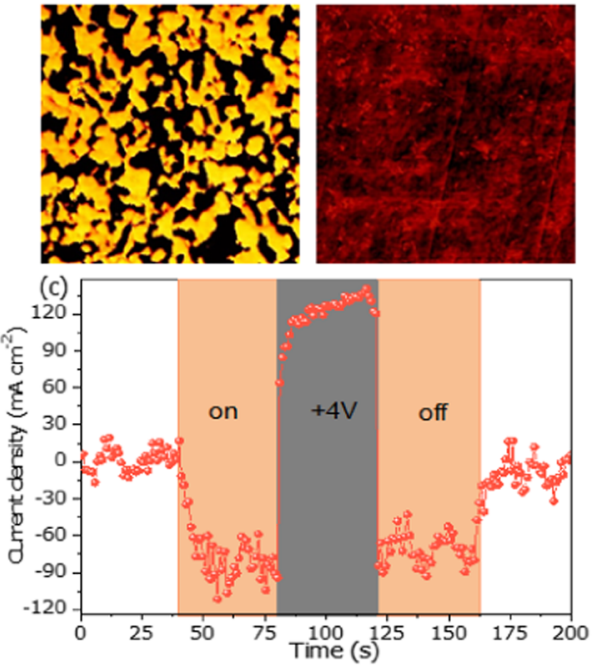

(f) Light and -4V (large area scan)
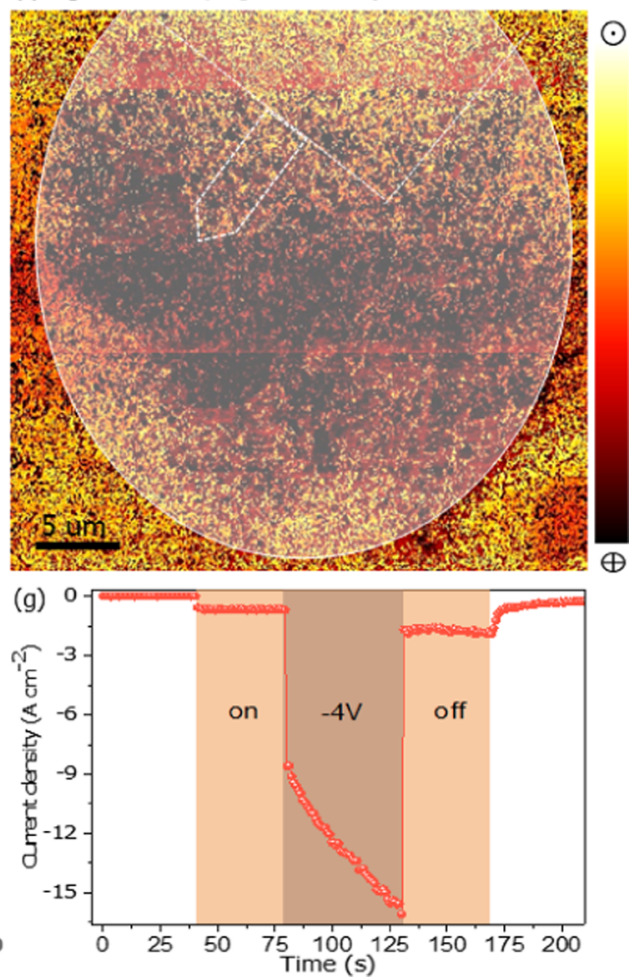

Figure 3. Cumulative effect of the light and applied electric field: (a, d) PFM phase images captured in a dark condition. Changes in the domain structure of the pristine area after cumulative poling under light (incident on a large sample area) and electric bias of (b) $+4 \mathrm{~V}$ and (e) $-4 \mathrm{~V}$ applied at a single point (marked in (a, d)) with a $30 \mathrm{~nm}$ AFM tip. (f) Large area scan to show the full-switched area under cumulative action of light and $-4 \mathrm{~V}$ for $40 \mathrm{~s}$ (white shaded area indicates light illumination area on the sample). Charge injection due to light-assisted electrical poling with a bias of $(\mathrm{c})+4 \mathrm{~V}$ and $(\mathrm{g})-4 \mathrm{~V}$. All measurements were carried out with the configuration, where $\mathrm{Cr}-\mathrm{Au}$ was the bottom electrode and the PFM tip acted as the top electrode.

further lowers the Schottky barrier and facilitates charge collection, as shown in Figure 2e. The phenomenon is also anticipated to have more excited carriers in the conduction band as a result of lowering the Schottky barrier, despite the negligible difference in the transmittance spectra of unpoled and poled samples (Figure 2f) corresponding to a $405 \mathrm{~nm}$ wavelength. Following this, two orders of magnitude modulation of the sample conductivity was observed as a function of laser intensity (laser spot size: $0.5 \mathrm{~mm}^{2}$ ) when measured macroscopically on KNBNNO sandwiched between indium tin oxide (ITO) electrodes (Figure 2g). Additionally, light-dependent (laser spot size: $0.5 \mathrm{~mm}^{2}$; power: $50 \mathrm{~mW}$; intensity: $10 \mathrm{~W} \mathrm{~cm}{ }^{-2}$ ) modulation of in-plane conductivity corresponding to applied voltages $( \pm 0.5$ to $\pm 4 \mathrm{~V})$ over a wide inter-electrode gap $(140 \mu \mathrm{m})$ was measured in a ferroelectric semiconductor field-effect transistor configuration ${ }^{45}$ and is shown in Figure $2 \mathrm{~h}$. The results indicate that the light acts as a virtual electrode for KNBNNO. Although the ratio of light and dark conductivities is remarkably high corresponding to all applied voltages, the in-plane current, as shown in Figure S4, in the Supporting Information is significantly low. However, the out-of-plane current was observed to be comparatively high in the same unpoled sample. To enhance the out-of-plane current output, the sample asymmetry was enhanced by poling it for 30 min in an electric field of $6 \mathrm{kV} \mathrm{mm}{ }^{-1}$. Although poling did not have much impact on in-plane conductivities, the observed light to dark conductivity ratio was significantly high (Figure $2 \mathrm{i}$ ) in the poled sample. This suggests that poled KNBNNO is an ideal material candidate for a monolithic two-terminal phototransistor/solaristor (MFSM configuration (ITOKNBNNO-ITO)). ${ }^{23}$ Comparing the unpoled and poled states of the same sample, in the former, the domains were randomly distributed in the ceramic sample, thus the net built-in filed was zero. The asymmetric behavior of the latter was introduced by the overall domain orientation, which induced a built-in field perpendicular to the sample surface.

Furthermore, light to dark conductivity ratios $\left(\sigma_{\text {Light }} / \sigma_{\text {Dark }}\right.$ Figure 2i) under applied voltages help in determining the voltage thresholds for optoelectric applications such as asymmetric rectification of AC bias $\left(\sigma_{\text {Light }} / \sigma_{\text {Dark }}<1\right)$, and for the use as a gating material for ferroelectric field-effect transistors $\left(\sigma_{\text {Light }} / \sigma_{\text {Dark }}<1\right)$ and photodetectors $\left(\sigma_{\text {Light }} / \sigma_{\text {Dark }}\right.$ $>1)$. The same mechanism helps in understanding the lightassisted ferroelectric poling. Since light acts as a virtual electrode with enhanced surface conductivity, the applied electric field modulates the photocurrent and results in a high charge injection over a broad area. This charge injection leads to a fast and ultralarge domain switching area (much larger than the electrical contact of the AFM tip), which could further be controlled by a laser spot size and the applied electric field. The domain switching behavior excluding the effect of charge injection has been studied elsewhere with time-dependent measurements. ${ }^{14}$ The difference of the light to dark ratios of the conductivity between the devices with in-plane and out-ofplane configurations may be attributed to different poling states (unpoled in Figure $2 \mathrm{~h}$ and poled in Figure $2 \mathrm{i}$ ) and different work functions of the electrodes (Ag in Figure $2 \mathrm{~h}$ and 

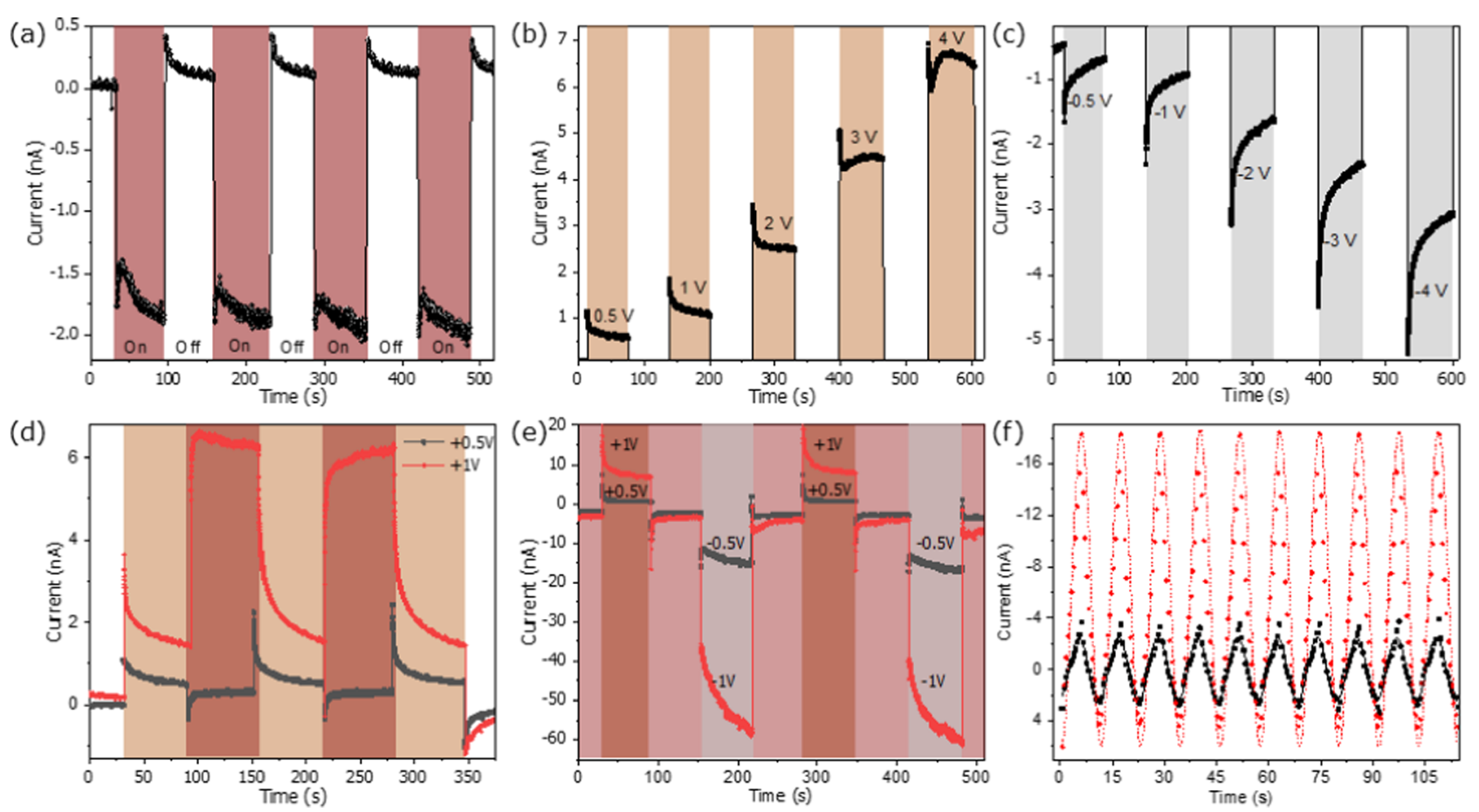

Figure 4. Modulation of the photocurrent and the electrical current: (a) photocurrent (without the electric field) and the electrical current corresponding to the applied (b) positive and (c) negative bias (measured in the dark) on a bulk-poled KNBNNO sample. Modulation of the electrical current with light due to the applied (d) positive bias of +0.5 and $+1 \mathrm{~V}$. (e) Modulation of the photocurrent by the applied negative and positive biases of \pm 0.5 and $\pm 1 \mathrm{~V}$. (f) Demonstration of asymmetric AC photoferroelectric rectification behavior. All measurements were carried out with the out-of-plane device configuration.

ITO in Figure 2i). ${ }^{46}$ These factors may affect the surface charge carrier distributions and thus domain dynamics.

\section{LIGHT-ASSISTED ELECTRICAL POLING}

Figure $3 a-c$ reveals the effect of light-triggered positive poling with $+4 \mathrm{~V}$, while the effect of light-assisted negative poling with $-4 \mathrm{~V}$ is displayed in Figure $3 \mathrm{~d}-\mathrm{g}$. The reproducibility of the results at two different locations is illustrated in the Supporting Information: Figures S5 and S6 for light-assisted positive poling, and Figures S7 and S8 for light-assisted negative poling. Initially, the laser was turned on for $40 \mathrm{~s}$ followed by the application of $+4 \mathrm{~V}$ (Figure $3 \mathrm{c}$ ) or $-4 \mathrm{~V}$ (Figure $3 \mathrm{~g}$ ) for $40 \mathrm{~s}$ at the center of the area (shown by a circle in Figure $3 \mathrm{a}, \mathrm{d}$ ) using a $30 \mathrm{~nm}$ AFM tip. Interestingly, the out-of-plane photocurrent was modulated in the opposite direction with nearly the same magnitude using $+4 \mathrm{~V}$, while the negative bias led to a modulation of two orders of magnitude in the same direction (Figure 3c,g). The growth time for $8000 \%$ modulation with negative bias was $\sim 1 \mathrm{~s}$, while the full-modulation of $16000 \%$ was achieved in around $100 \mathrm{~s}$, which is similar to the photocurrent rise time in the absence of an applied electric field, as shown in Figure S3a in the Supporting Information. This indicates that a combination of laser intensity and AC bias can be used to obtain asymmetric half-wave rectification, which was demonstrated macroscopically, as shown in Figure 4 and the discussions below. The post poling shrinkage of the out-of-plane bright domains in Figure $3 \mathrm{~b}$ was found to be fully reversible by the application of $-4 \mathrm{~V}$ for $10 \mathrm{~s}$ at the same spot in the dark, as shown in Figure S9 in the Supporting Information. It shows that the charge injected by the cumulative action of the light and electric field can be reversed by applying an electric field alone in the opposite direction through the AFM tip placed at the same spot. The reversal of domains (see Figure S9 in the Supporting Information) leads to the possibility of the creation of connected charged channels across the domain walls ${ }^{47}$ due to light-triggered single-point electrical poling. The same channels could be utilized for the domain reversal if a higher current than the modulated photocurrent is applied in the opposite direction. The same is true for light-assisted negative poling, but it was only confirmed macroscopically (Figure 4) due to the limitation of the maximum voltage required to completely reverse the domain states that could not be applied using the AFM system on such a large switched area, as shown in Figure 3f. Besides, it was revealed that light-assisted positive poling could lead to optoelectric domain wall velocities of $72 \mathrm{~nm} \mathrm{~s}^{-1}$ (the average change in the distance between the two domains in Figure $3 \mathrm{~b}$ ), which could further be increased to $30000 \mathrm{~nm} \mathrm{~s}^{-1}$ using lightassisted negative poling (estimated as the minor radius of the ellipse (equivalent to the switched area) in Figure 3f). Lightassisted negative poling also results in ultralarge domain area switching (450 000 times in contrast to the electrode (AFM tip) area), which could further be controlled by an applied voltage (as shown in Figure $\mathrm{S} 10$ in the Supporting Information) and laser spot size (as shown in Figure S11 in the Supporting Information). Figure S10 shows that the switched area was extended equally in all directions due to an increase in the applied voltage. A similar extension of the switched area with an increasing applied voltage (in the dark) has been reported for several other ferroelectrics and is attributed to the pinning potential and domain wall creep. ${ }^{48,49}$ The domain wall velocity in such a scenario is given by (v) $\sim v_{0} \mathrm{e}^{\left[-E_{\mathrm{a}} / E\right] \mu}$, where $E_{\mathrm{a}}$ is the activation field, $E$ is the applied electric field, ${ }^{48,50}$ and $\mu$ is an exponent governing the nature of the pinning potential and dimensionality of the 
wall. ${ }^{48,50}$ Lou et al. suggested a statistical model that explains the same phenomena in bulk ferroelectrics. ${ }^{51}$ Optoelectrically tuned in-plane conductivity and corresponding charge migration (Figure 2h) modulates the pinning potential, resulting in fast domain wall moment for low applied electric fields in $\mathrm{KNBNNO}$ in comparison to the reported higher electric fields in several well-known ferroelectrics such as $\mathrm{Pb}(\mathrm{Zr0} 2 \mathrm{Ti0} .8) \mathrm{O}_{3}{ }^{48}$ poly(vinylidene-fluoride-trifluorethylene), ${ }^{52}$ and $\mathrm{BaTiO}_{3}{ }^{50}$ Post poling stability of the switched domains was studied at two different locations (locations 2 and 3) for 60 and $120 \mathrm{~min}$, as shown in Figures $S 12$ and $S 13$ in the Supporting Information. No changes were observed in optoelectrically poled domains, whereas, only optically poled domains return to their original position in $17 \mathrm{~min} .{ }^{14}$ The robustness of optoelectrically poled domains makes them ideal for optoelectric modulators.

\section{APPLICATIONS AND CONCLUSIONS}

To demonstrate the potential applications of KNBNNO, a device was prepared using $120 \mu \mathrm{m}$ thick KNBNNO sandwiched between two $200 \mathrm{~nm}$ thick ITO electrodes. In this arrangement, KNBNNO works as a monolithic twoterminal photodetector or photoswitch and a ferroelectric light-effect transistor ${ }^{40}$ or a solaristor, ${ }^{23}$ where one electrode acts as the source while the other as the drain and light exposure as a virtual gate corresponding to those in conventional semiconductor transistors. KNBNNO was initially poled electrically $\left(6 \mathrm{kV} \mathrm{mm}^{-1}\right.$ for $\left.30 \mathrm{~min}\right)$ to achieve higher asymmetry and built-in fields. It is to be noted that the sample was very sensitive to light and its polarization changed even in ambient lighting conditions. The specific state of polarization can, to some extent, be ascertained by the magnitude of the measured photocurrent. Figure S14a,b in the Supporting Information shows the comparison of the photocurrent from the same sample for two different states of the polarization. A comparison of the photocurrent (in the absence of an external electric field) and electrical currents corresponding to an applied positive and negative bias (measured in the dark) is presented in Figure $4 a-c$, respectively. The same sample was used to illustrate the modulation of the electrical current (Figure $4 \mathrm{~d}$ ) and photocurrent (Figure 4e). Interestingly, the electrical current corresponding to $+0.5 \mathrm{~V}$ is suppressed by the presence of light, while the current corresponding to $+1 \mathrm{~V}$ and higher voltages is enhanced in the positive direction, as shown in Figure $4 \mathrm{~d}$. In principle, the resultant current should be the cumulative result of the electrical $(+0.7 \mathrm{nA})$ and photocurrent $(-1.8 \mathrm{nA})$, which is not the case here. The resultant of the applied electric field and built-in field helps in controlling the direction of the photocurrent. The sample's asymmetry directs the photocurrent in a negative direction, while the applied positive bias guides it in a positive direction. The final outcome is governed by the equilibrium state of ferroelectric domains (as also found at the nanoscale in Figure $3 a, b)$. With the higher applied voltage of $+1 \mathrm{~V}$, the photocurrent is also modulated in a positive direction, and the resultant electrical current is enhanced. This could also be complemented with the out-ofplane light to dark conductivity ratio $(<1)$ corresponding to $+0.5 \mathrm{~V}$ for the poled sample (see Figure $2 \mathrm{i}$ ). The suppression of the electrical current corresponding to $+0.5 \mathrm{~V}$ due to light illumination was confirmed for two different states of polarization, where the obtained photocurrents with the same sample were different, as shown in Figure S14 in the
Supporting Information. In both conditions, a voltage of +0.5 $\mathrm{V}$ results in an increase in the electrical current in a positive direction. However, the electrical current decreases to +0.2 and $+0.5 \mathrm{nA}$, corresponding to the states of polarization upon light illumination of the sample (see Figure S14a,b in the Supporting Information). On the contrary, the electrical current with higher voltages is increased in the respective directions by the presence of light. Due to this reason, a bias of $0.5 \mathrm{~V}$ was chosen to demonstrate asymmetric AC modulation of the dark current (Figure 4f). The AC dark current for an applied voltage of $1 \mathrm{~V}$ (peak to peak) can be modeled by fitting eq 1 (shown by a black line in Figure 4e). The modulated photocurrent for the same applied voltage is modeled using eq 2 (shown by a red line in Figure 4e). Similarly, several other functions could be designed to obtain the desired output using the presented photoferroelectric function generator.

$$
\begin{aligned}
& y=y_{0}+A \cdot \sin \left(\pi\left(x-x_{c}\right) / w\right) \\
& y=y_{0}+A \cdot\left(\sin \left(\pi\left(x-x_{c}\right) / w\right)\right)^{2}
\end{aligned}
$$

Interestingly, the cumulative action of the light and electric field for voltages higher than $0.5 \mathrm{~V}$ modulates the individually obtained current. The difference in the modulated magnitude for the applied positive and negative bias can be explained on the basis of $E_{\mathrm{Net}}$ due to the built-in field. The same is also supported by the dark and light conductivity ratio. The current modulation was found to be consistent and reproducible, irrespective of the process (voltage applied before turning the laser on or laser turned on before applying voltage, as shown in Figure S15 in the Supporting Information). This voltagedependent modulation of the photocurrent makes KNBNNO suitable for a photodetector or self-powered two-terminal phototransistor. In addition, several orders magnitude change in the photocurrent by application of small voltages suggests that KNBNNO's photovoltaic performance can be significantly tuned by applying small voltages. Though KNBNNO shows untypical $I-V$ (current-voltage) characteristics and in some cases the electrical output is lower than the traditional existing devices, we expect that the presented findings and the proof of concepts for using ferroelectrics as a monolithic photoferroelectric function generator/modulator, asymmetric AC rectifier, and light-effect transistor are likely to motivate the scientific community for the development of improved optoferroelectric devices used in next-generation applications.

\section{ASSOCIATED CONTENT}

\section{SI Supporting Information}

The Supporting Information is available free of charge at https://pubs.acs.org/doi/10.1021/acsaelm.0c00497.

Detailed experimental section; material and device quality; reversible optical control of ferroelectric domains; light assisted ferroelectric poling; post poling domain reversal; controlling of the poled area by the electric field and laser spot size; stability test; macroscopic current modulation (PDF)

\section{AUTHOR INFORMATION}

\section{Corresponding Authors}

Gaurav Vats - School of Materials Science and Engineering, University of New South Wales, Sydney, NSW 2052, Australia; Email: g.vats@unswalumni.com 
Jan Seidel - School of Materials Science and Engineering, University of New South Wales, Sydney, NSW 2052, Australia; ○ orcid.org/0000-0003-2814-3241; Email: jan.seidel@ unsw.edu.au

Yang Bai - Microelectronics Research Unit, Faculty of Information Technology and Electrical Engineering, University of Oulu, FI-90014 Oulu, Finland; (c) orcid.org/0000-00020861-2410; Email: yang.bai@oulu.fi

\section{Authors \\ Jani Peräntie - Microelectronics Research Unit, Faculty of Information Technology and Electrical Engineering, University of Oulu, FI-90014 Oulu, Finland \\ Jaakko Palosaari - Microelectronics Research Unit, Faculty of Information Technology and Electrical Engineering, University of Oulu, FI-90014 Oulu, Finland \\ Jari Juuti - Microelectronics Research Unit, Faculty of Information Technology and Electrical Engineering, University of Oulu, FI-90014 Oulu, Finland}

Complete contact information is available at:

https://pubs.acs.org/10.1021/acsaelm.0c00497

\section{Notes}

The authors declare no competing financial interest.

\section{ACKNOWLEDGMENTS}

The authors acknowledge support from the Australian Research Council (ARC) through Discovery Grants. G.V. acknowledges the financial support from Tiina and Antti Herlin Foundation, Finland. J.P. acknowledges the funding by the Academy of Finland (grant number 298409). Y.B. would like to acknowledge the joint funding by the University of Oulu and Academy of Finland profiling action "Ubiquitous wireless sensor systems" (grant number 24302332). The authors also acknowledge the Centre for Material Analysis of the University of Oulu for the use of their facilities and for the fabrication of the electrodes. G.V. carried out the nanoscale and macroscopic characterizations. J.P. performed a part of the macroscopic measurements. J.P. helped to fabricate the samples. Y.B. fabricated the samples and carried out the macroscopic characterizations. All authors co-wrote the paper.

\section{REFERENCES}

(1) Paillard, C.; Bai, X.; Infante, I. C.; Guennou, M.; Geneste, G.; Alexe, M.; Kreisel, J.; Dkhil, B. Photovoltaics with Ferroelectrics: Current Status and Beyond. Adv. Mater. 2016, 28, 5153-5168.

(2) Pandey, R.; Vats, G.; Yun, J.; Bowen, C. R.; Ho-Baillie, A.; Seidel, J.; Butler, K. T.; Seok, S. I. Mutual Insight on Ferroelectrics and Hybrid Halide Perovskites: A Platform for Future Multifunctional Energy Conversion. Adv. Mater. 2019, 31, No. 1807376.

(3) Fan, Z.; Sun, K.; Wang, J. Perovskites for photovoltaics: a combined review of organic-inorganic halide perovskites and ferroelectric oxide perovskites. J. Mater. Chem. A 2015, 3, 1880918828.

(4) Butler, K. T.; Frost, J. M.; Walsh, A. Ferroelectric materials for solar energy conversion: photoferroics revisited. Energy Environ. Sci. 2015, 8, 838-848.

(5) Burger, A. M.; Agarwal, R.; Aprelev, A.; Schruba, E.; GutierrezPerez, A.; Fridkin, V. M.; Spanier, J. E. Direct observation of shift and ballistic photovoltaic currents. Sci. Adv. 2019, 5, No. eaau5588.

(6) Tan, L. Z.; Zheng, F.; Young, S. M.; Wang, F.; Liu, S.; Rappe, A. M. Shift current bulk photovoltaic effect in polar materials-hybrid and oxide perovskites and beyond. npj Comput. Mater. 2016, 2, No. 16026.
(7) Young, S. M.; Rappe, A. M. First Principles Calculation of the Shift Current Photovoltaic Effect in Ferroelectrics. Phys. Rev. Lett. 2012, 109, No. 116601.

(8) Seidel, J.; Fu, D.; Yang, S.; Alarcon-Llado, E.; Wu, J.; Ramesh, R.; Ager, Joel W., III Efficient Photovoltaic Current Generation at Ferroelectric Domain Walls. Phys. Rev. Lett. 2011, 107, No. 126805.

(9) Yang, S. Y.; Seidel, J.; Byrnes, S. J.; Shafer, P.; Yang, C.; Rossell, M. D.; Yu, P.; Chu, Y.; Scott, J. F.; Ager, J. W.; Martin, L. W.; Ramesh, R. Above-bandgap voltages from ferroelectric photovoltaic devices. Nat. Nanotechnol. 2010, 5, 143-147.

(10) Spanier, J. E.; Fridkin, V. M.; Rappe, A. M.; Akbashev, A. R.; Polemi, A.; Qi, Y.; Gu, Z.; Young, S. M.; Hawley, C. J.; Imbrenda, D.; Xiao, G.; Bennett-Jackson, A. L.; Johnson, C. L. Power conversion efficiency exceeding the Shockley-Queisser limit in a ferroelectric insulator (vol 10, pg 611, 2016). Nat. Photonics 2016, 10, 688.

(11) Alexe, M.; Hesse, D. Tip-enhanced photovoltaic effects in bismuth ferrite. Nat. Commun. 2011, 2, No. 256.

(12) Rubio-Marcos, F.; Del Campo, A.; Marchet, P.; Fernandez, J. F. Ferroelectric domain wall motion induced by polarized light. Nat. Commun. 2015, 6, No. 6594.

(13) Rubio-Marcos, F.; Ochoa, D. A.; Del Campo, A.; Garcia, M. A.; Castro, G. R.; Fernandez, J. F.; Garcia, J. E. Reversible optical control of macroscopic polarization in ferroelectrics. Nat. Photonics 2018, 12, 29.

(14) Vats, G.; Bai, Y.; Zhang, D.; Juuti, J.; Seidel, J. Optical Control of Ferroelectric Domains: Nanoscale Insight into Macroscopic Observations. Adv. Opt. Mater. 2019, 7, No. 1800858.

(15) Yang, M.; Alexe, M. Light-Induced Reversible Control of Ferroelectric Polarization in $\mathrm{BiFeO} 3$. Adv. Mater. 2018, 30, No. 1704908.

(16) Páez-Margarit, D.; Rubio-Marcos, F.; Ochoa, D. A.; Del Campo, A.; Fernandez, J. F.; Garcia, J. E. Light-Induced Capacitance Tunability in Ferroelectric Crystals. ACS Appl. Mater. Interfaces 2018, 10, 21804-21807.

(17) Bai, Y.; Vats, G.; Seidel, J.; Jantunen, H.; Juuti, J. Boosting Photovoltaic Output of Ferroelectric Ceramics by Optoelectric Control of Domains. Adv. Mater. 2018, 30, No. 1803821.

(18) Chanthbouala, A.; Garcia, V.; Cherifi, R. O.; Bouzehouane, K.; Fusil, S.; Moya, X.; Xavier, S.; Yamada, H.; Deranlot, C.; Mathur, N. D.; Bibes, M.; Barthelemy, A.; Grollier, J. A ferroelectric memristor. Nat. Mater. 2012, 11, 860-864.

(19) Liou, Y.-D.; Chiu, Y.; Hart, R. T.; Kuo, C.; Huang, Y.; Wu, Y.; Chopdekar, R. V.; Liu, H.; Tanaka, A.; Chen, C.; Chang, C.; Tjeng, L. H.; Cao, Y.; Nagarajan, V.; Chu, Y.; Chen, Y.; Yang, J. Deterministic optical control of room temperature multiferroicity in $\mathrm{BiFeO} 3$ thin films. Nat. Mater. 2019, 18, 580.

(20) Wang, X.; Wang, P.; Wang, J.; Hu, W.; Zhou, X.; Guo, N.; Huang, H.; Sun, S.; Shen, H.; Lin, T.; Tang, M.; Liao, L.; Jiang, A.; Sun, J.; Meng, X.; Chen, X.; Lu, W.; Chu, J. Ultrasensitive and Broadband MoS2 Photodetector Driven by Ferroelectrics. Adv. Mater. 2015, 27, 6575 .

(21) Abel, S.; Eltes, F.; Ortmann, J. E.; Messner, A.; Castera, P.; Wagner, T.; Urbonas, D.; Rosa, A.; Gutierrez, A. M.; Tulli, D.; Ma, P.; Baeuerle, B.; Josten, A.; Heni, W.; Caimi, D.; Czornomaz, L.; Demkov, A. A.; Leuthold, J.; Sanchis, P.; Fompeyrine, J. Large Pockels effect in micro-and nanostructured barium titanate integrated on silicon. Nat. Mater. 2019, 18, 42.

(22) Choi, T.; Lee, S.; Choi, Y. J.; Kiryukhin, V.; Cheong, S.-W. Switchable Ferroelectric Diode and Photovoltaic Effect in BiFeO3. Science 2009, 324, 63-66.

(23) Pérez-Tomás, A.; Lima, A.; Billon, Q.; Shirley, I.; Catalan, G.; Lira-Cantu, M. A Solar Transistor and Photoferroelectric Memory. Adv. Funct. Mater. 2018, 28, No. 1707099.

(24) Hu, W. J.; Wang, Z.; Yu, W.; Wu, T. Optically controlled electroresistance and electrically controlled photovoltage in ferroelectric tunnel junctions. Nat. Commun. 2016, 7, No. 10808.

(25) Tsymbal, E. Y.; Gruverman, A. Beyond the barrier. Nat. Mater. 2013, 12, 602-604. 
(26) Bai, Y.; Palosaari, J.; Tofel, P.; Juuti, J. A single-material multisource energy harvester, multi-functional sensor and integrated harvester-sensor system - Demonstration of concept. Energy Technol. 2020, No. 2000461.

(27) Vats, G.; Peräntie, J.; Juuti, J.; Seidel, J.; Bai, Y. Coalition of thermo-opto-electric effects in ferroelectrics for enhanced cyclic multienergy conversion. Energy Technol. 2020, No. 2000500.

(28) Jentjens, L.; Peithmann, K.; Maier, K.; Steigerwald, H.; Jungk, $\mathrm{T}$. Radiation-damage-assisted ferroelectric domain structuring in magnesium-doped lithium niobate. Appl. Phys. B 2009, 95, 441-445.

(29) Valdivia, C. E.; Sones, C. L.; Mailis, S.; Mills, J. D.; Eason, R. $\mathrm{W}$. Ultrashort-pulse optically-assisted domain engineering in lithium niobate. Ferroelectrics 2006, 340, 75-82.

(30) Eggert, H. A.; Kalkum, F.; Wengler, M. C.; Heinemeyer, U.; Buse, K. Light-assisted generation of tailored ferroelectric domain structures. Ferroelectrics 2006, 340, 63-67.

(31) Ying, Y. J.; Valdivia, C. E.; Sones, C. L.; Eason, R. W.; Mailis, S. Latent light-assisted poling of LiNbO3. Opt. Express 2009, 17, 18681-18692.

(32) Shur, V.; Rumyantsev, E.; Nikolaeva, E.; Shishkin, E.; Fursov, D.; Batchko, R.; Eyres, L.; Fejer, M.; Byer, R. Nanoscale backswitched domain patterning in lithium niobate. Appl. Phys. Lett. 2000, 76, 143145 .

(33) Steigerwald, H.; Ying, Y. J.; Eason, R. W.; Buse, K.; Mailis, S.; Soergel, E. Direct writing of ferroelectric domains on the $\mathrm{x}$ - and $\mathrm{y}$ faces of lithium niobate using a continuous wave ultraviolet laser. Appl. Phys. Lett. 2011, 98, No. 062902.

(34) Kuznetsov, D. K.; Shur, V. Y.; Negashev, S. A.; Lobov, A. I.; Pelegov, D. V.; Shishkin, E. I.; Zelenovskiy, P. S.; Platonov, V. V.; Ivanov, M. G.; Osipov, V. V. Formation of Nano-Scale Domain Structures in Lithium Niobate Using High-Intensity Laser Irradiation. Ferroelectrics 2008, 373, 133-138.

(35) Bai, Y.; Tofel, P.; Palosaari, J.; Jantunen, H.; Juuti, J. A Game Changer: A Multifunctional Perovskite Exhibiting Giant Ferroelectricity and Narrow Bandgap with Potential Application in a Truly Monolithic Multienergy Harvester or Sensor. Adv. Mater. 2017, 29, No. 1700767.

(36) Catalan, G.; Seidel, J.; Ramesh, R.; Scott, J. F. Domain wall nanoelectronics. Rev. Mod. Phys. 2012, 84, 119-156.

(37) Seidel, J.; Eng, L. M. Shedding light on nanoscale ferroelectrics. Curr. Appl. Phys. 2014, 14, 1083-1091.

(38) Kufer, D.; Konstantatos, G. Photo-FETs: Phototransistors Enabled by 2D and OD Nanomaterials. ACS Photonics 2016, 3, 2197-2210.

(39) de Arquer, F. P. G.; Armin, A.; Meredith, P.; Sargent, E. H. Solution-processed semiconductors for next-generation photodetectors. Nat. Rev. Mater. 2017, 2, No. 16100.

(40) Marmon, J. K.; Rai, S. C.; Wang, K.; Zhou, W.; Zhang, Y. LightEffect Transistor (LET) with Multiple Independent Gating Controls for Optical Logic Gates and Optical Amplification. Front. Phys. 2016, 4,8 .

(41) Bai, Y.; Xiang, H.; Jantunen, H.; Juuti, J. Multi-functional perovskites - an investigation of compositional and processing influence on microstructure, dielectric and ferroelectric properties. Eur. Phys. J.: Spec. Top. 2019, 228, 1555-1573.

(42) Damjanovic, D. Ferroelectric, dielectric and piezoelectric properties of ferroelectric thin films and ceramics. Rep. Prog. Phys. 1998, 61, 1267-1324.

(43) Borkar, H.; Rao, V.; Tomar, M.; Gupta, V.; Scott, J. F.; Kumar, A. Experimental evidence of electronic polarization in a family of photo-ferroelectrics. RSC Adv. 2017, 7, 12842-12855.

(44) Belinicher, V.; sturman, B. The Relation between Shift and Ballistic Currents in the Theory of Photogalvanic Effect. Ferroelectrics 1988, 83, 29-34.

(45) Si, M.; Saha, A. K.; Gao, S.; Qiu, G.; Qin, J.; Duan, Y.; Jian, J.; Niu, C.; Wang, H.; Wu, W.; Gupta, S. K.; Ye, P. D. A ferroelectric semiconductor field-effect transistor. Nat. Electron. 2019, 2, 580-586.

(46) Würfel, P.; Würfel, U. Physics of Solar Cells: From Basic Principles to Advanced Concepts; Wiley-VCH: Weinheim, 2016.
(47) Seidel, J. Domain Walls as Nanoscale Functional Elements. J. Phys. Chem. Lett. 2012, 3, 2905-2909.

(48) Tybell, T.; Paruch, P.; Giamarchi, T.; Triscone, J. Domain wall creep in epitaxial ferroelectric $\mathrm{Pb}(\mathrm{Zr} 0.2 \mathrm{Ti} 0.8) \mathrm{O}-3$ thin films. Phys. Rev. Lett. 2002, 89, No. 097601.

(49) Liu, Q. D.; Huber, J. E. Creep in ferroelectrics due to unipolar electrical loading. J. Eur. Ceram. Soc. 2006, 26, 2799-2806.

(50) Pertsev, N. A.; Petraru, A.; Kohlstedt, H.; Waser, R.; Bdikin, I. K.; Kiselev, D.; Kholkin, A. L. Dynamics of ferroelectric nanodomains in $\mathrm{BaTiO} 3$ epitaxial thin films via piezoresponse force microscopy. Nanotechnology 2008, 19, No. 375703.

(51) Lou, X. J. Statistical switching kinetics of ferroelectrics. J. Phys.: Condens. Matter 2009, 21, No. 012207.

(52) Xiao, Z.; Poddar, S.; Ducharme, S.; Hong, X. Domain wall roughness and creep in nanoscale crystalline ferroelectric polymers. Appl. Phys. Lett. 2013, 103, No. 112903. 